Trends and pattern of white-tailed deer

(Odocoileus virginianus) in mayan villages,

Campeche, Mexico

\title{
Patrones y tendencias de uso del venado cola blanca (Odocoileus virginianus) en comunidades mayas, Campeche, México
}

\author{
Oscar Gustavo Retana-Guiascón ${ }^{1 *}$, Lucia Guadalupe Martínez-Pech¹, Graciela Niño-Gómez ${ }^{1}$, Eloy Victoria-Chan ${ }^{1}$, \\ Ángeles Cruz-Mass ${ }^{1}$ y Alejandro Uc-Piña ${ }^{1}$
}

\begin{abstract}
${ }^{1}$ Centro de Estudios en Desarrollo Sustentable y Aprovechamiento de la Vida Silvestre, Universidad Autónoma de Campeche. Avenida Agustín Melgar s/n. San Francisco de Campeche 24039. Campeche, México. E-mail: retana1967@yahoo.com.mx, ogretana@uacam.mx (OGRG), lumarp_86@hotmail.com (LGMP), tazmania2408@gmail.com (GNG), fucionax@hotmail.com (EVC), angeles282@hotmail.com (ACM), alexopulus12@hotmail.com (AUP).

${ }^{*}$ Corresponding author
\end{abstract}

Introduction: For some indigenous societies of Mexico, white-tailed deer (Odocoileus virginianus) has played and still plays a decisive role in terms of its cultural and practical significance. In this sense, the objective of this study was to obtain information concerning the diverse ways that Mayan populations of Campeche State using the white-tailed deer with the aim of detecting patterns and trends of use on this species.

Methods: Fieldwork (2009-2012) was carried out in four rural communities, Nunkiní and Sahcabchén located in the northern portion of Campeche, and Pich and Chencoh located in the central part of the state. Interviews $(n=160)$ were performed to record the uses and values people assign to the wildlife in each community. Using the relative importance index (IIRE) and the similarity index (IS), a comparison was made between communities regarding white-tailed deer values and uses.

Results: In the four Mayan communities, uses of white-tailed deer ( $O$. virgininianus) can be classified in eight categories: 1) food, 2) medical, 3) furrier, 4) tool, 5) ornamental, 6) trade, 7) pets, and 8) mythical. The use of deer as food showed an IS $>0.8$ in four communities. Skin trade was recorded differently with Pitch having a higher value of importance with respect to the communities of Chencoh, Sahacabchen and Nunkiní, where IS held in a range from 0.4 to 0.6. The most significant differences were obtained for skin trade showing a similar index between Sahacabchen and Nunkini (IS > 0.6 ) while lacking commercial value in Chencoh and Pich $(I S<0.1)$.

Discussion and conclusions: The recognized patterns of use of white-tailed deer recognized are related to the historical characteristics of utilization and management of natural resources of the Mayan communities, where agriculture is the predominant economical activity and hunting is an opportunistic and occasional practice for subsistence. This pattern of use has remained relatively stable and the practices and categories of use are similar among Mayan populations despite the distance between them $(\sim 100 \mathrm{~km})$. However, communities in Northern Campeche (Nunkiní and Sahcabchen) showed a growing tendency to trade the skin with respect to communities of Central Campeche (Chencóh and Pich) where the traditional pattern of use remained essentially for food, ornamental and household use of skin, with a tendency towards implementing hunting in legally established wildlife management units (UMA).

Key words: cervidae; indigenous knowledge; multiple use; subsistence hunting; Yucatan peninsula.

Introducción: Para varios grupos indígenas de México el venado cola blanca (Odocoileus virginianus), ha jugado y aun juega un papel determinante en términos de su importancia cultural y material. En este sentido, el objetivo de este estudio fue obtener información relativa a las formas de utilización del venado por poblaciones mayas del estado de Campeche, con el propósito de establecer un patrón de tendencias de uso de esta especie.

Metodología: Durante 2009 a 2012 se trabajó con cuatro comunidades Mayas; Nunkini y Sahcabchen (ubicadas al norte del estado de Campeche), Pich y Chencoh (localizadas en el centro de la entidad). A partir de entrevistas 
estructuradas ( $n=160$ ) se registró el valor de uso del venado en cada comunidad. Mediante el Índice de Importancia Relativa (IIRE) y el Índice de Similitud (IS), se realizó una correlación entre las comunidades respecto a las formas y valor de uso del venado.

Resultados: En las cuatro comunidades, el uso del venado cola blanca se clasifica en ocho categorías: 1) alimento, 2) medicina, 3) peletero, 4) herramienta, 5) ornamento, 6) comercio, 7) mascota y 8) mítico. El uso alimentario del venado mostro un IS $>0.8$ en las cuatro comunidades, en tanto el uso peletero registro diferencias ya que en Pich tuvo un alto valor de importancia con respecto a las comunidades de Chencoh, Sahacabchen y Nunkini en donde el IS se mantuvo en un rango de 0.4 a 0.6. Las diferencias más significativas se obtuvieron para el uso comercial de la piel mostrando una tendencia similiar entre Sahacabchen y Nunkini (IS = $>0.6)$, en tanto para Chencoh y Pich dicha categoria de uso es nula $(I S=0.0)$.

Discusión y conclusiones: El patrón de uso reconocido para el venado cola blanca está relacionado a las características históricas de aprovechamiento y manejo de recursos de las comunidades mayas en donde la agricultura ha sido la actividad económica predominante y la cacería una práctica ocasional y oportunista para subsistencia. Este patrón de uso se ha mantenido relativamente estable, pues aun entre poblaciones mayas alejadas 100 kilómetros las formas de aprovechamiento de $O$. virginianus son similares, tanto en términos de las categorías de uso como en el valor que tiene el venado a nivel intra e inter-comunitario. No obstante, en Nunkini y Sahcabchen se registra una tendencia en los últimos 10 años a comercializar la piel del venado en contraste con Pich y Chencoh, en donde se mantiene el patrón de uso tradicional esencialmente como alimento, ornato y piel para uso doméstico y donde existe una tendencia hacia el aprovechamiento cinegético bajo el sistema de unidades de manejo de vida silvestre (UMA).

Palabras clave: cervidae; conocimiento indígena; cacería de subsistencia; Odocoileus virginianus; península de Yucatán; uso múltiple.

\section{Introducción}

A finales del Pleistoceno, hace aproximadamente 35 mil años, el venado cola blanca (Odocoileus virginianus) se distribuía desde Sonora hasta la Península de Yucatán, como lo confirman los registros de restos óseos encontrados en el Lago de Chapala, Jalisco, el cerro de Tlapacoya, Estado de México, cueva de Loltún, Yucatán, y Villaflores, Chiapas (Álvarez y Polaco 1983; Lorenzo y Mirambell 1999; Arroyo y Polaco 2003; Montellano y Carbot 2009; Gómez y Carbot 2012). Durante la transición del Pleistoceno tardío al Holoceno (de los 23 a los 9 mil años), se desencadenaron una serie de cambios climáticos que repercutieron, en parte, en la extinción de la megafauna, así como en una nueva estructura de la vegetación y composición faunística (Grayson 1989). Por lo cual, los grupos de cazadores-recolectores se readaptaron a un nuevo modo de vida y de obtención de recursos. La caza se volvió más diversificada a través de un nomadismo cíclico que les permitió aprovechar estacionalmente la variedad de recursos. El venado es una de las especies faunísticas más importantes como alimento, materia prima y medio de expresión cultural. Este hecho se muestra en las pinturas rupestres localizadas en el conjunto de barrancas y cuevas en la sierra de Lerdo, Durango y San Francisco de la Sierra, Baja California Sur (Casado 2015). Los grabados más antiguos en México de los distintos mamíferos, como los de venados, se realizaron hace 7,500 años (Casado 2015).

El ambiente más estable a lo largo del ciclo anual, permitió a los grupos humanos comenzar a seleccionar y utilizar plantas y animales, hecho que conduciría paulatinamente a la agricultura (hace 7,000 a 4,500 años). El desarrollo de la agricultura permitió incrementar la densidad de la población humana, lo que además propicio el inicio de las primeras comunidades sedentarias. Este cambio en los patrones de actividad de los humanos marco el parteaguas para el desarrollo de las sociedades mesoamericanas. La fauna silvestre no perdería valor, por el contrario, 
sería un elemento esencial de su cultura (Retana 2006). En el México prehispánico, el venado cola blanca (O. virginianus) fue un recurso esencial y su piel se empleó para la escritura de los códices, así como la elaboración de diversos productos como huaraches, morrales y cestos (Galindo y Weber 1998). Es una de las especies animales con alta presencia en los aspectos cosmogónicos, formando parte del calendario ritual o tonalpohualli con el nominativo "Mázatl" en náhuatl. Representó al séptimo día, asociado a Tláloc. En los códices mayas y se relaciona con deidades de la lluvia y la fertilidad, como la asociación con IxChel, patrona de la cacería y medicina (Montoliu 1977; Chávez 2012).

El venado forma parte de las estrategias de uso múltiple y manejo diversificado de la fauna silvestre que les ha permitido a las comunidades mayas satisfacer gran parte de sus requerimientos materiales y culturales (Toledo et al. 2008; Sarukhán et al. 2009). El objetivo de este estudio fue obtener información relativa a las formas de utilización del venado cola blanca por poblaciones mayas del estado de Campeche, con el propósito de establecer un patrón de uso actual y determinar las tendencias que puedan afectar o favorecer su conservación a nivel local.

\section{Material y métodos}

Área de estudio. Se trabajó con cuatro comunidades mayas del estado de Campeche; Nunkini y San Antonio Sahcabchen (se denominará Sahcabchen de aquí en adelante). Ambas comunidades se localizan al norte del estado, en la región conocida culturalmente como Camino Real. Las comunidades Pich y Chencoh están en la zona central del estado que se conoce como región de los Chenes (Figura 1; Gómez 2001). Los principales criterios para la selección de las comunidades fueron: 1) Trabajar con dos comunidades contiguas de la región norte y dos de la región centro. 2) Una distancia geográfica mínima de $100 \mathrm{~km}$ entre las comunidades del norte y las del centro. 3) Las comunidades reportaran el aprovechamiento de fauna silvestre como una práctica tradicional para alimentación familiar, zooterapia y materia prima a nivel local.

La comunidad de Nunkiní ( $\left.20^{\circ} 24^{\prime} 05^{\prime \prime} \mathrm{N},-90^{\circ} 08^{\prime} 56^{\prime \prime} \mathrm{O}\right)$ cuenta con una población total de 5,859 habitantes y una superficie forestal ejidal de 57 mil hectáreas (ha). Su economía se basa principalmente en la agricultura de maíz y el cultivo de hortalizas. La apicultura y la manufactura de diversos materiales de palma de jipi (Carludovica palmata) y guano (Sabal spp.) son actividades secundarias. La ganadería es básicamente para autoconsumo (INEGI 2011, 2015).

La comunidad de Sahcabchen $\left(20^{\circ} 18^{\prime} 30^{\prime \prime} \mathrm{N},-90^{\circ} 08^{\prime} 11^{\prime \prime} \mathrm{O}\right)$ tiene una población de 1,858 habitantes y una superficie forestal ejidal de 9 mil ha. Su economía se basa en la agricultura tradicional con semillas criollas para autoconsumo y siembra de hortalizas, así como la apicultura y cría de ganados ovino y bovino. Son actividades secundarias la elaboración de hamacas, sillas y cortinas, actualmente se está retomando el uso tradicional de fibras vegetales de lengua de vaca (Sansevieria hyacinthoides) y el chelém (Agave angustifolia; INEGI 2011, 2015).

La comunidad de Pich ( $19^{\circ} 29^{\prime} 11^{\prime \prime} \mathrm{N}$ y $-90^{\circ} 07^{\prime} 05^{\prime \prime} \mathrm{O}$ ) cuenta con una población de 1,756 habitantes y una superficie forestal ejidal de 58,127 ha. Su principal actividad económica es la agricultura. Son actividades secundarias la apicultura, ganadería y aprovechamiento forestal, contando con una ampliación forestal superior a las 46 mil hectáreas (Figura 1). Algunos pobladores ejercen oficios de asociados a servicios (INEGI 2011, 2015). 


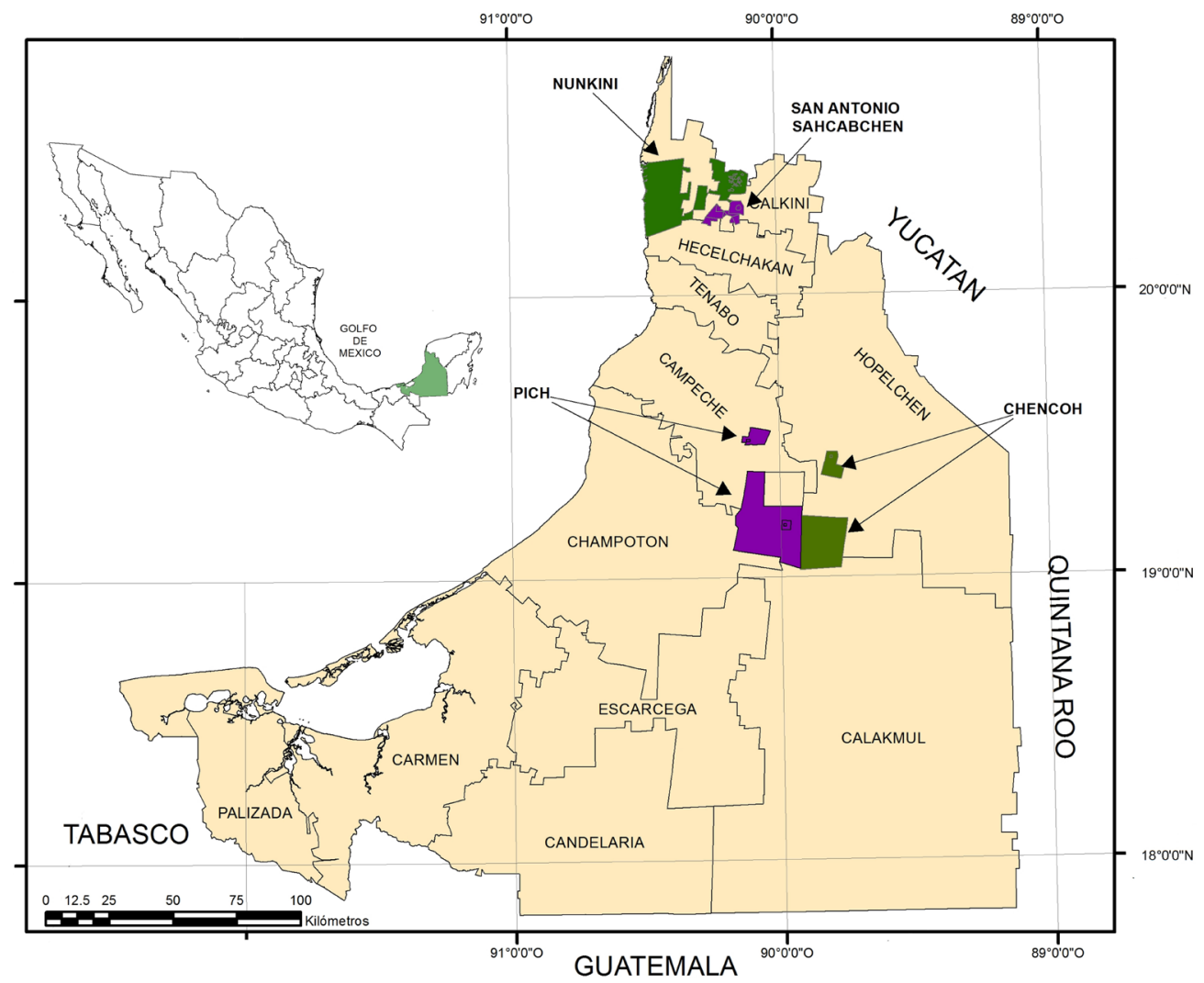

Figura 1. Localización de las comunidades mayas y representación de su superficie ejidal y ampliación forestal de cada una.

La comunidad maya de Chencoh ( $19^{\circ} 25^{\prime} 24^{\prime \prime} \mathrm{N}$ y $-89^{\circ} 48^{\prime} 26^{\prime \prime} \mathrm{O}$ ) cuenta con una población total de 477 habitantes y una superficie forestal ejidal de 38,253 ha. Su economía se basa en la agricultura de temporal, maíz y frijol. Son actividades secundarias la apicultura, cría de ovinos, aprovechamiento forestal y trabajos asalariados (INEGI 2011, 2015). Estas dos últimas comunidades durante el periodo de estudio realizaron el aprovechamiento legal de fauna cinegética en sus ampliaciones forestales mediante su registro como Unidad de Manejo y Aprovechamiento Sustentable de la Vida Silvestre (UMA).

Dada su cercanía a la costa $(<35 \mathrm{~km})$ en la región donde se ubican las comunidades de Nunkini y Sahcabchen, ambas a 10 msnm, existen tres tipos de vegetación; selva baja caducifolia, selva mediana subcaducifolia y sabana (Flores y Espejel 1994). En las comunidades de Pich $(112 \mathrm{~m})$ y Chencoh $(174 \mathrm{~m})$ se registran predominantemente dos tipos de vegetación; la selva mediana subperennifolia y selva baja perennifolia (inundable). Esta última se caracteriza por encontrarse en las zonas bajas con drenaje deficiente, que se inundan en la época de lluvias y permanece así por más de cinco meses por lo que son comunes las asociaciones de palo de tinte (Haematoxylum campechianum; Flores y Sánchez 2010). El clima predominante para las cuatro comunidades es el cálido subhúmedo (Aw) con una temperatura media anual de 26 a $27^{\circ} \mathrm{C}$, la temporada de lluvias queda localizada a los meses de mayo a octubre con una precipitación media anual de 1,000 a 1,100 mm (Mendoza y Kú 2010).

En términos de su componente mastofaunístico, Campeche forma parte de la unidad biogeográfica denominada Provincia Biótica Península de Yucatán (Vázquez 
y Arita 2010), por lo que su afinidad es principalmente neotropical. Para la entidad se reportan 111 especies de mamíferos terrestres lo que equivale al $22.37 \%$ de representatividad a nivel nacional (Rámirez-Pulido et al. 2014). Los artiodáctilos constituyen el $4.5 \%$ del total de especies registradas para el estado (Guzmán et al. 2013). En lo que respecta a la Familia Cervidae, en el territorio campechano habitan tres especies; el temazate rojo (Mazama temama), el temazate café (M. pandora), y el venado cola blanca (O. virginianus). De las 14 subespecies reconocidas de venado cola blanca para México, tres se reportan con distribución para Campeche: O. v. thomasi, O. v. yucatanensis y O. v. truei. (Mandujano et al. 2010).

Conocimiento y uso del venado cola blanca. La información relativa al conocimiento y uso que los pobladores mayas poseen en torno al venado cola blanca, se obtuvo durante 2009 a 2012 por medio de un proyecto sobre valoración de uso de la fauna silvestre en las comunidades Mayas antes señaladas financiado por Fomix Campeche. Se empleó el método de valoración participativa (Chambers 1994), aplicando las técnicas de entrevistas estructuradas y no estructuradas. La entrevista no estructurada (no estandarizada), se realizó para tener el primer acercamiento con los habitantes de cada comunidad, como medio y vínculo de confianza con los pobladores de cada comunidad, a fin de que supieran de qué se trataba el estudio y establecer el horario de visita y personas interesadas en participar. La entrevista estructurada (estandarizada), se condujo posteriormente utilizando un formato preestablecido con preguntas cerradas para registrar la importancia y frecuencia de aquellas especies animales, los productos de éstas que son aprovechadas y el valor de uso. Se aplicaron 40 entrevistas estandarizadas en cada comunidad ( $n=160$ ), a personas adultas elegidas al azar ( $>25$ años) y que contaran con más de 20 años de residencia en la zona. Como parte de las personas entrevistadas se incluyeron a los cazadores que aceptaron ser entrevistados, asimismo las entrevistas individuales que terminaron siendo familiares se consideraron como una sola entrevista.

Con el fin de evaluar la importancia comunitaria del venado de acuerdo a las categorías de uso que se registran en cada comunidad, se calculó el Índice de Valor de Importancia Relativa (IIRE): $\mathrm{nc} / \mathrm{N}$ x 100. Donde $n c$ es el número de informantes que citan a la especie dentro de la categoría de uso y $N$ el número total de informantes.

Para conocer el grado de semejanza entre las comunidades mayas en relación al uso del venado cola blanca se estimó el índice de similitud (Cosió 2007; Frankfort y León 2009), mediante la siguiente formula: IS=1-( $\triangle P R / 100)$. Donde IS es el índice de similitud y $\triangle P R$ es la diferencia de IIRE entre comunidades. Cuando el IS tiende a 1 hay mayor similitud en cuanto a valor de uso del venado cola blanca entre las comunidades mayas. Los datos obtenidos se capturaron en una matriz en formato MS-Excel y fueron procesados mediante estadística descriptiva, especialmente análisis de frecuencias y porcentajes utilizando el paquete estadístico SPSS versión 12.0 para Windows ${ }^{\circledR}$ (SPSS Inc. 2003).

\section{Resultados}

El venado cola blanca (O. virgininianus) se aprovecha en ocho categorías de uso que a continuación se detallan.

1. Alimento. El venado tiene un alto valor de uso como alimento en las cuatro comunidades mayas estudiadas. Los valores del IS muestran que es una especie con el mismo valor de importancia y predilección independientemente de la ubicación 
geográfica y/o condición socio-ambiental de cada comunidad (Figura 2). El consumo de la carne se realiza de diversas formas; pipian, asado, ceviche, tzik (salpicón) y ché chak (caldo).

2. Medicina. Los valores de IS muestran una mayor similitud de uso medicinal entre las comunidades del norte del estado (IS $\geq 0.6$ ) en contraste con las comunidades del sur (IS $\leq$ 0.4). En las comunidades de Nunkini y Sahcabchen se usa la grasa para tratar tres afecciones respiratorias (bronquitis, tos, asma) y una reumática (dolor de huesos). La ceniza que resulta de quemar la cola del venado se usa para tratar el dolor de oídos. En tanto que en las comunidades de Pich y Chencoh, la grasa se usa únicamente para tratar la tos y dolor de huesos.

3. Peletero. En cuanto al valor de uso peletero, se documentó que aún se sigue aprovechando la piel del venado para elaborar diversos productos como: sandalias, correas, fajas, sillas, mecedoras, fundas de machete y bolsos. En las comunidades de Nunkini (IS = 0.49), Sahcabchen (IS = 0.40) y Chencoh (IS =0.42), los valores fueron proporcionalmente similares en cuanto al uso de las pieles de manera doméstica. En la comunidad de Pich (IS > 0.9), se observa un alto reconocimiento de la población en torno al valor peletero del venado.

4. Comercio. En las comunidades de Nunkini y Sahcabchen se registró comercio de pieles secas a precios que van de los 200 a 500 pesos (13 a 33 USD) por pieza según su tamaño. En Pich y Chencoh el uso comercial solo es a baja escala y localmente, siendo mayor su aprovechamiento comunitario para elaborar diversos productos. En Nunkini y Sahcabchen el comercio de carne de venado tiene un precio de 85 a 100 pesos por kilo (5 a 7 USD), aunque no es una práctica común. El precio depende si es vendida dentro o fuera de las comunidades.

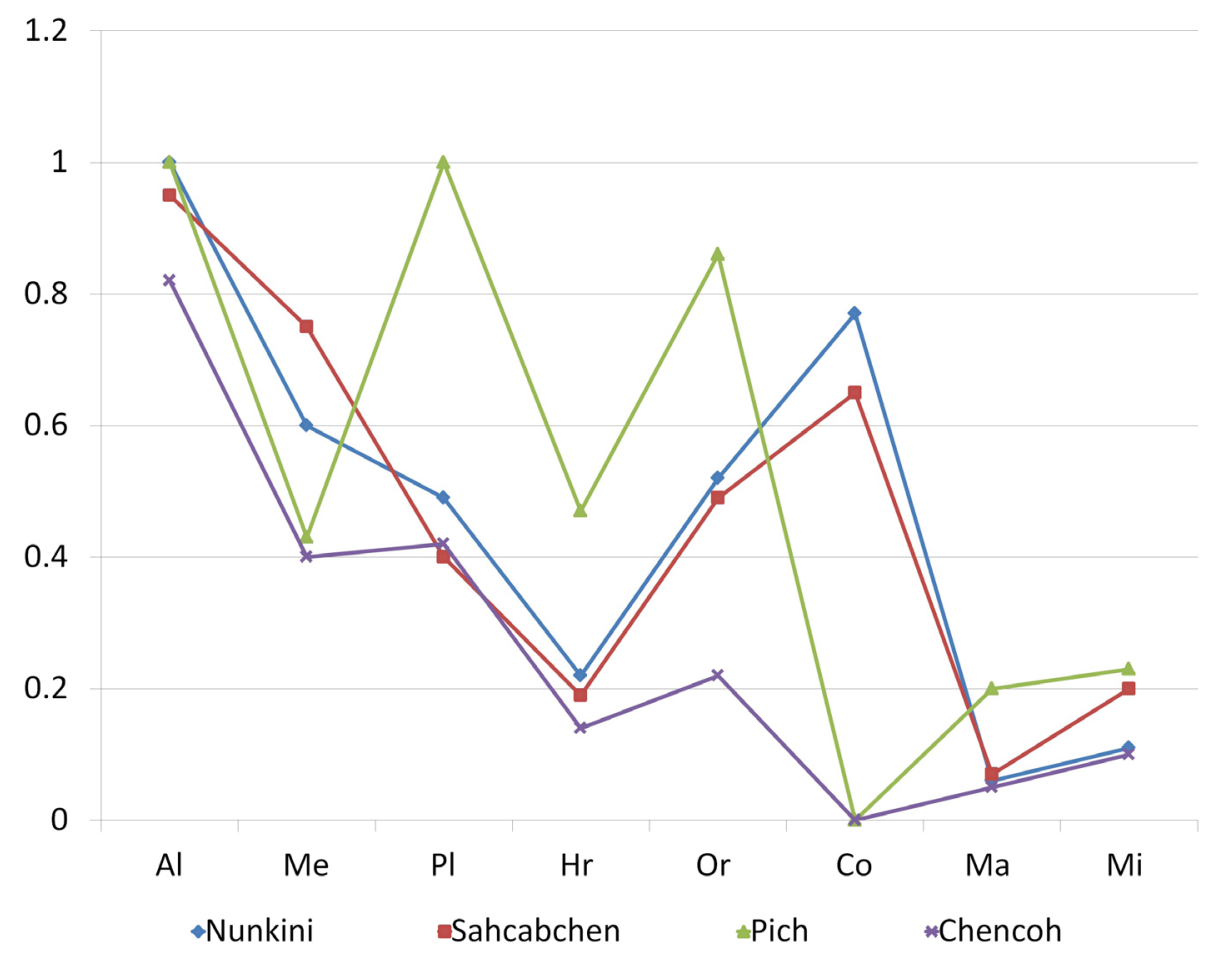

Figura 2. Índice de similitud del venado cola blanca (Odocoileus virginianus) entre comunidad maya por categoría de uso. $\mathrm{Al}=$ alimento, $\mathrm{Me}=$ medicina, $\mathrm{PI}=$ peletero, $\mathrm{Hr}=$ herramienta, $\mathrm{Or}=$ ornamento, $\mathrm{Co}=$ comercio, $\mathrm{Ma}=$ mascota $\mathrm{Mi}=$ Mítico. Pich $=$ verde Chencoh $=$ morado, Nunkini $=$ azul y Sahcabchen $=$ rojo 
5. Mascota. En las cuatro comunidades se registró la venta ocasional de cervatillos para criarlos como mascota.

6. Herramienta. En las cuatro comunidades se aprovechan las astas como percheros, quitar las hojas (brácteas), desgranar las mazorcas, elaborar mangos para navajas y cuchillos. Las patas se usan para elaborar mangos de cuartas (fuetes), destapadores y llaveros.

7. Ornamento. En las comunidades de Nunkini (IS $=0.52$ ) y Sahcabchen (IS = 0.49) el uso ornamental es más frecuente, lo que se relacionó con la mayor actividad comercial que se realiza en estas comunidades.

8. Mítico. El venado cola blanca en lengua maya es conocido con el nombre genérico de "Kéej", nombre onomatopéyico en atribución al sonido que produce con la nariz cuando advierte peligro (Chable 2000). En el ámbito mítico en las cuatro comunidades se obtuvo que se usa la pata del venado para golpear nueve veces la rodilla de los niños pequeños para que aprendan a caminar rápido. Asimismo, se documentó que cuando se caza un venado no se debe tirar, regalar o cocinar el hígado junto con las demás vísceras, debido a que esto hará que el arma pierda el aire (potencia de tiro) y no se logrará matar otros animales. Destaca la mención, de que en caso de encontrarse la piedra "tunich" en el estómago del venado es usada como amuleto para tener buena suerte en la caza. Según los valores del IS $(<0.24)$, se muestra que los aspectos míticos en torno al venado son similares en las comunidades, no obstante estos son del dominio solamente de algunos pobladores (cazadores principalmente).

\section{Discusión}

Los índices de similitud más altos $(0.52,1-0)$ se encuentran en general en la comunidad de Pich y los más bajos en la de Chencoh $(0.26,0.82-0)$, ambas localizadas en la zona central del estado que se conoce como región de los Chenes. Las comunidades del norte Sahcabchen y Nunkini tienen índices muy similares. Esto se debe a que en estas poblaciones mayas se registra la misma tendencia de uso del venado.

En todas las comunidades se obtuvo que el índice por alimentación fue el más alto $(0.94,1-0.82)$, los que implica que la principal motivo por la que se realiza la cacería es por el producto cárnico, quedando los otros siete como secundarios a excepción del peletero en la comunidad de Pich $(I S=1)$. El valor más bajo $(0.095$, $0.2-0.05)$ fue para el de mascota, seguido del mítico $(0.16,0.23-0.1)$. Destaca el hecho de que en las cuatro comunidades todos los pobladores entrevistados ubican al venado cola blanca como la especie animal con mayor importancia alimenticia a nivel intra e intercomunitaria, esto se ha mantenido desde tiempos prehispánicos (Götz 2011, 2012, 2014; Masson y Peraza 2008, Montero 2009). Por el contrario, el uso mítico registro un valor muy bajo habiendo sido tan importante en la cosmología maya. El uso que más variación presento fue el de comercio desde IS $=0.77$ a IS = 0 . Registrando particularmente esta tendencia para las comunidades del norte con un valor de similitud proporcionales, si se considera la diferencia en el tamaño de las comunidades.

Patrón ytendencias de uso del venado cola blanca. Nuestros resultados corresponden con otros estudios, como el realizado en la comunidad Maya "El Remate" localizada al norte de Campeche. En "El Remate", el venado se ubica como uno de los recursos 
alimenticios con mayor preferencia con respecto a la 35 especies faunísticas que son aprovechadas por los pobladores de esa comunidad (Méndez y Montiel 2007). Sin embargo, pueden existir variaciones en el patrón de uso con respecto a comunidades mayas dedicadas a la pesca, como La Isla, en Campeche, en donde su actividad económica principal se sustenta en el aprovechamiento de especies marinas.

El aprovechamiento del venado en las ocho categorías de uso identificadas entre las comunidades mayas estudiadas, permite reconocer que existe un patrón de uso de este cérvido entre poblaciones mayas. Patrón que posiblemente se fue constituyendo desde la época prehispánica, ya que de acuerdo a las investigaciones arqueozoológicas llevadas a cabo en distintos sitios del área Maya. El venado aparece como una de las especies preferidas en los asentamientos mayas durante el clásico tardío (Emery 2003; Montero 2009). El venado cola blanca fue la especie animal más frecuente encontrada en los basureros asociados con las zonas habitacionales del Petén, al sur de la península, y zona norte de la Península de Yucatán (Götz 2014). Restos de venados subadultos en las muestras arqueofaunísticas correspondientes al periodo postclásico en la antigua ciudad Maya de Mayapán, se ha interpretado como una posible evidencia de manejo de venados en áreas agroforestales controladas (Masson y Peraza 2008).

La tendencia a comercializar la piel de venado en la comunidad Nunkini se debe a la demanda que han generado compradores foráneos, así como a la venta local que se produce cada año debido a la celebración de su carnaval. Las pieles de venado se utilizan como parte de la vestimenta de las personas que participaran en el llamado carnaval de "osos". En las comunidades de Pich y Chencoh, el uso de la piel es más de tipo doméstico, esto coincide con lo reportado para las poblaciones de Tankuché y El Remate ubicadas al norte del estado de Campeche, México, en donde se reporta que la piel se usa para fabricar productos de talabartería como fundas para machetes y forros para monturas (Méndez y Montiel 2007; León y Montiel 2008).

El uso mítico del venado forma parte de las manifestaciones culturales de las comunidades mayas, destacando la búsqueda del "tunich". Se trata de una piedra bezoar (calculo intestinal) que suele encontrarse generalmente en el reticulum, que corresponde a una de las cámaras del estómago, la cual es un amuleto muy cotizado por los cazadores (Mandujano y Rico 1991).

El patrón de uso reconocido para el venado cola blanca está relacionado a las característicashistóricas deaprovechamientoymanejo de recursos delascomunidades mayas en donde la agricultura ha sido la actividad económica predominante y la cacería una práctica ocasional y oportunista para subsistencia. En este contexto, el registro de ocho tipos de uso de $O$. virginianus es una medida diagnóstica de la importancia y el aprovechamiento de esta especie entre las poblaciones mayas del estado de Campeche como recurso estratégico a nivel local, materia prima y producto de intercambio o comercio. El patrón de uso se ha mantenido relativamente estable, pues entre poblaciones mayas distanciadas en Campeche a 100 kilómetros y en las cuales no registró que tuvieran contacto, las formas de aprovechamiento de $O$. virginianus son similares, tanto en términos de las categorías de uso como en el valor que tiene el venado a nivel intra e inter-comunitario. No obstante, en las comunidades ubicadas en el norte se registra una variación en cuanto a la tendencia de cazar venados para comercializar su piel. Por lo cual es fundamental evaluar el impacto que está generando esta actividad comercial sobre las poblaciones de venado cola blanca que habitan en la región norte del estado, así como buscar alternativas de permitan 
regular y legalizar el aprovechamiento de esta especie animal para favorecer su conservación a nivel local y regional.

\section{Agradecimientos}

Agradecemos a C. Lorenzo, S. Mandujano y R. Reyna-Hurtado sus acertadas observaciones y recomendaciones realizadas a este manuscrito.

\section{Literatura citada}

Alvarez, T., y O. J. Polaco. 1983. Restos de moluscos y mamíferos cuaternarios procedentes de Loltúm, Yucatán. Cuaderno de Trabajo No. 26. Departamento de Prehistoria, Instituto Nacional de Antropología e Historia. Ciudad de México, México.

Arroyo, C. J., y O. J. Polaco. 2003. Caves and the Pleistocene vertebrate paleontology of México. Pp. 273-291 en Ice age cave faunas of North America (Schubert, B.W., J. I. Mead, y R. W. Graham, eds.). Indiana University Press y Denver Museum of Nature and Science. Denver, EE. UU.

CAsado, L. M. 2015. El Arte Rupestre en México. Arqueología Mexicana 61:1-82.

ChablÉ, H. E. 2000. Máayáh Táan. Cuaderno de enseñanza del idioma. Casa de la Cultura, Hopelchén. Campeche, México.

Chambers, R. 1994. The Origins and Practice of Participatory Rural Apprasial. World Development 22:953-969.

Chávez, G. J. 2012. Significados del venado-sol en la cosmovisión maya. Un atisbo a la mitología e historia oral mayence. Editorial Académica Española. Madrid, España.

Cosıo, B. A. 2007. Conocimiento y comparación del uso de la Fauna Silvestre en dos Comunidades Ejidales del Municipio de Hueytamalco, Puebla, México. Tesis de Maestría. Instituto de Ecología A. C. Xalapa, México.

EmerY, K. F. 2003. The noble beast: status and differential access to animals in the Maya world. World Archaeology 34:498-515.

Flores, J. S., Y I. C. EsPejel. 1994. Tipos de vegetación de la península de Yucatán. Etnoflora Yucatanense. Fascículo 3. Universidad Autónoma de Yucatán. Mérida, México.

Flores, J. S., y M. C. SÁnchez. 2010. Diversidad florística. Pp. 201-2013 en La Biodiversidad en Campeche: Estudio de Estado (Villalobos, G., y J. Mendoza, coords.). Comisiòn Nacional para el Conocimiento y Uso de la Biodiversidad - Gobierno del Estado de Campeche - Universidad Autónoma de Campeche - El Colegio de la Frontera Sur (ECOSUR). Unidad Campeche. Campeche, México.

Frankfort, N. C., y A. León. 2009. Social Statistics for a Diverse Society. 5th ed. Pine Forge Press. Oxnard, EE. UU.

Galindo, C., y M. Weber. 1998. El Venado de la Sierra Madre Occidental. Ecología, Manejo y Conservación. Comisiòn Nacional para el Conocimiento y Uso de la BiodiversidadEdiciones Culturales SA DICUSA. Ciudad de México, México.

García, E. 1981. Modificaciones al Sistemas de Clasificación Climática de Koppen. Tercera Edición. Ciudad de México, México.

Gómez J. J. 2001. El Estado de Campeche. Pp. 41-52 en Los Mayas de Campeche frente a la modernización (Gómez de Silva, J., ed.). Gobierno del Estado de Campeche - 19972003. Campeche, México.

Gómez, P. L., Y G. СаRвot. 2012. Contribución al estudio de los megamamíferos del Pleistoceno Tardío del municipio de Villaflores, Chiapas, México. Lacandonia 6:31-41.

González, D. 1997. Algunas consideraciones sobre la cacería de subsistencia tradicional 
de venado en algunos ejidos mayas integrantes del plan piloto forestal. V Simposio sobre Venados de México. Universiadad Nacional Autònoma de México, Distrito Federal, México.

Götz, M. C. 2011. Una mirada zooarqueológica a los modos alimenticios de los mayas de las Tierras Bajas del norte. Pp. 89-109 en Identidades y cultura material en la región maya (Hernández, H. A., y M. Pool, eds.). Universidad Autónoma de Yucatán. Mérida, México.

Götz, M.C. 2012. Critical evaluation of the sustainability of Prehispanic Maya agroecosystems: implications of hunting and animal domestication in the Northern Maya Lowlands. Pp. 477-486 en The Archaeology of Yucatan: New Directions and Data, (Stanton, T., ed.). British Archaeological Reports International Series. Oxford, EE.UU.

Götz, M. C. 2014. La alimentación de los Mayas Prehispánicos vista desde la zooarqueología. Anales de Antropología 48:167-199.

Grayson, D. K. 1989. The chronology of North American late Pleistocene extinctions. Journal of Archeological Science 16:153-165.

Guzmán, S. D., O. G. Retana, y J. Cú. 2013. Lista de los Mamíferos Terrestres del Estado de Campeche, México. Acta Zoológica Mexicana (n. s.) 29:105-128.

INEGI. 2011. Censo de Población y Vivienda (2010). Panorama sociodemográfico de Campeche. Instituto Nacional de Estadística y Geografía. Aguscalientes, México.

INEGI. 2015. Instituto Nacional de Estadística y Geografía (2010). Principales resultados por localidad 2010 (ITER) . Consulta en línea: junio-2015.

León, P., Y S. Montiel. 2008. Wild meat use and traditional hunting practices in a rural Mayan community of the Yucatan Peninsula. Human Ecology 36:249-257.

Lorenzo, J. L., Y L. Mirambell. 1999. The Inhabitants of Mexico During the Upper Pleistocene. Pp. 482-496 en Ice Age People of North America, (Bonnichsen, R., y K. Turnmire, eds.). Oregon State University Press. Corvallis, EE.UU.

Mandujano, S., y V. Rico. 1991. Hunting, use, and knowledge of the biology of the whitetailed deer (Odocoileus virginianus Hays) by the maya of central Yucatan, Mexico. Journal of Ethnobioogy 11:175-183.

Mandujano, S., C. Delfín, y S. Gallina. 2010. Comparison of geographic distribution models of white-tailed deer Odocoileus virginianus (Zimmermann, 1780) subspecies in Mexico: biological and management implications. Therya 1:41-68.

Masson, M., y C. Peraza. 2008. Animal use at the Postclassic Maya center of Mayapán. Quaternary International 191:170-183.

Méndez, C. F., y S. Montiel. 2007. Diagnóstico preliminar de la Fauna y Flora Silvestre utilizada por la población Maya de dos comunidades costeras de Campeche, México. Universidad y Ciencia 23:127-139.

Mendoza, V. J., y V. M. Kú. 2010. Clima. Pp. 16-19 en La Biodiversidad en Campeche: Estudio de Estado (G. Villalobos, y J. Mendoza, coords.). Comisión Nacional para el Conocimiento y Uso de la Biodiversidad - Gobierno del Estado de Campeche Universidad Autónoma de Campeche -El Colegio de la Frontera Sur (ECOSUR) Unidad Campeche. Campeche, México.

Montellano, B. M., y G. CаRвot. 2009. Presencia de Odocoileus (Artiodactyla, Cervidae) en el Pleistoceno de Chiapas, México. Pp. 291-298 en 60 años de la Colección Nacional de Mamíferos del Instituto de Biología de la UNAM. Aportaciones al Conocimiento y Conservación de los Mamíferos de México (Cervantes, F., Y. Ortelano, y J. Vargas, Eds.). Instituto de Biología, Universidad Nacional Autónoma de México. Ciudad de México, Mexico. 
Montero, L. C. 2009. Sacrifice and feasting among the classic maya elite, and the importance of the with-tailed deer: is there a regional pattern? Journal of Historical and European Studies 2:53-68.

Montiel, S., L. M. Arias, y F. Dickinson. 1999. La cacería tradicional en el norte de Yucatán: una práctica comunitaria. Universidad Autónoma de Chapingo. Revista de Geografía Agrícola 29:43-52.

Montiel, O. S., y L. ARias. 2008. La caceria tradicional en el Mayab contemporaneo: una mirada desde la ecología humana. Avance y Perspectiva 1:21-27.

Ramírez-Pulido, J., N. González, A. Gardner y J. Arroyo. 2014. List of Recent Land Mammals of Mexico, Special Publications, Museum of Texas Tech University 63:1-69.

Retana, O. G. 2006. Fauna Silvestre de México. Aspectos Históricos de su Gestión y Conservación. Ciencia y Tecnología . Fondo de Cultura Económica. Ciudad de México, México.

Sarukhán, J., P. Koleff, J. Carabias, J. Soberón, R. Dirzo, J. Llorente-Bousquets, G. Halffter.R. González, March, A. Mohar, S. Anta, y J. De la Maza. 2009. Capital natural de México. Síntesis: actual, evaluación y perspectivas de sustentabilidad. Comisión Nacional para el Uso de la Biodiversidad. Ciudad de México, México.

Statistics Package for the Social Sciences (SPSS). 2003. Versión 12.0 for Windows. SPSS Inc. Chicago, EE. UU.

Toledo, V., N. Barrera, E. García, y P. Alarcón. 2008. Uso Múltiple y Biodiversidad entre los Mayas Yucatecos. México. Interciencia 33:345-362.

VÁzquez, D. E., y H. Arita. 2010. The Yucatan peninsula: biogeographical history 65 million years in the making. Ecography 33:212-219.

Summited: July 20, 2015

Review: September 2, 2015

Accepted: September 25, 2015

Associated editor: Rafael Reyna 
